Check for updates

Cite this: Chem. Sci., 2019, 10, 6120

๑ All publication charges for this article have been paid for by the Royal Society of Chemistry

Received 3rd April 2019

Accepted 12th May 2019

DOI: $10.1039 / c 9 s c 01648 a$

rsc.li/chemical-science

\title{
Direct triplet sensitization of oligothiophene by quantum dots $\dagger$
}

\author{
Zihao Xu, (D) a Tao Jin, ${ }^{a}$ Yiming Huang, ${ }^{b}$ Karimulla Mulla, ${ }^{b}$ \\ Francesco A. Evangelista, (D) *a Eilaf Egap (D) *b and Tianquan Lian (D) *a
}

\begin{abstract}
Effective sensitization of triplet states is essential to many applications, including triplet-triplet annihilation based photon upconversion schemes. This work demonstrates successful triplet sensitization of a CdSe quantum dot (QD)-bound oligothiophene carboxylic acid (T6). Transient absorption spectroscopy provides direct evidence of Dexter-type triplet energy transfer from the QD to the acceptor without populating the singlet excited state or charge transfer intermediates. Analysis of T6 concentration dependent triplet formation kinetics shows that the intrinsic triplet energy transfer rate in $1: 1$ QD-T6 complexes is $0.077 \mathrm{~ns}^{-1}$ and the apparent transfer rate and efficiency can be improved by increasing the acceptor binding strength. This work demonstrates a new class of triplet acceptor molecules for QDbased upconversion systems that are more stable and tunable than the extensively studied polyacenes.
\end{abstract}

\section{Introduction}

Triplet excitons in organic materials exhibit long lifetime, extended diffusion length and low-lying energy levels, ${ }^{1-3}$ and have found promising applications in photon upconversion. ${ }^{4}$ For example, an optical upconversion layer on a solar cell can capture sub-bandgap photons and emit above bandgap photons, ${ }^{5}$ increasing the efficiency of the conventional singlejunction devices beyond the Shockley-Queisser limit. ${ }^{6}$ Various strategies for improving the overall efficiency of upconversion systems have been developed. ${ }^{7-10}$ In recent years, quantum dot (QD) sensitized upconversion systems ${ }^{11-14}$ have attracted intense interest as a versatile and promising approach because of their large absorption coefficient, ${ }^{15}$ small singlet-to-triplet energy gap and fast intersystem crossing (ISC) rate, ${ }^{\mathbf{1 6}}$ and tunable band gap and band alignment. ${ }^{17}$

In a typical photon-upconversion system, triplet excitons are generated through a sensitizer that undergoes intersystem crossing from an excited singlet state to a triplet state. This process is followed by a sequential triplet energy transfer first to the transmitter then to the emitter. The latter, can undergo triplet-triplet-annihilation (TTA) and emit a higher energy photon.,11,13,18-22 Thus, the efficiency of a typical sensitizeremitter-based upconversion system can be represented by the following equation: ${ }^{4}$

${ }^{a}$ Department of Chemistry, Emory University, 1515 Dickey Dr, Atlanta, GA, 30322 USA. E-mail: francesco.evangelista@emory.edu; tlian@emory.edu

${ }^{b}$ Department of Materials Science and NanoEngineering, Department of Chemical and Biomolecular Engineering, Rice University, 6100 Main St, Houston, TX, 77005, USA. E-mail: ee30@rice.edu

† Electronic supplementary information (ESI) available. See DOI: 10.1039/c9sc01648a

$$
\Phi_{\mathrm{UC}}=\Phi_{\mathrm{ISC}} \Phi_{\mathrm{TET}} \Phi_{\mathrm{TTA}} \Phi_{\mathrm{FL}}
$$

The overall upconversion efficiency $\left(\Phi_{\mathrm{UC}}\right)$ is the product of efficiencies in each step involved, namely the ISC of the sensitizer $\left(\Phi_{\text {ISC }}\right)$, the triplet energy transfer $(\text { TET })^{23}$ from the sensitizer to transmitter to emitter $\left(\Phi_{\mathrm{TET}}\right)$, TTA of the emitter $\left(\Phi_{\text {TTA }}\right)$, and the emitter's fluorescence $\left(\Phi_{\mathrm{FL}}\right)$. For a specific sensitizer and emitter, $\Phi_{\mathrm{ISC}}, \Phi_{\mathrm{TTA}}$ and $\Phi_{\mathrm{FL}}$ are determined by the material's properties. A promising area for performance improvement is the design of $\mathrm{QD} /$ transmitter complexes to enable efficient TET. $^{24}$

In most reported QD sensitized upconversion systems, the transmitter/emitter molecules are solely limited to acenes and their derivatives, which have limited structural and energetic tunability and poor stability. ${ }^{11,13,18-22,25}$ Oligothiophenes have wide range of tunability in energetics and molecular structure, ${ }^{26}$ making them a very desirable class of triplet acceptors/ transmitters in QD-organic hybrid TTA upconversion systems. Although an example of oligothiophene phosphonic acid grafted to cadmium selenide (CdSe) QDs was previously reported, ${ }^{27}$ the photoluminescence quenching was attributed to charge transfer and TET was not observed. We hypothesize that with appropriate design of the energetics of QDs and oligothiophene acceptors, efficient TET transfer can be facilitated while competing single energy and charge transfer pathways may be suppressed (Fig. 1).

\section{Results and discussion}

In this work, we demonstrate for the first time a successful Dexter-type TET from CdSe QDs to carboxylic acid functionalized oligothiophene $\quad\left(3^{\prime \prime \prime}, 4^{\prime \prime}\right.$-dihexyl-[2,2' $: 5^{\prime}, 2^{\prime \prime}: 5^{\prime \prime}, 2^{\prime \prime \prime}: 5^{\prime \prime \prime}, 2^{\prime \prime \prime \prime}: 5^{\prime \prime \prime \prime}, 2^{\prime \prime \prime \prime \prime}$ - 


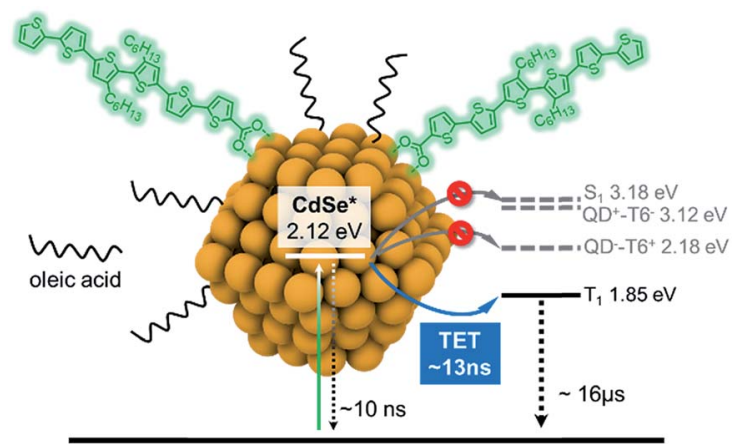

Fig. 1 Cartoon of QD-T6 complexes showing the energetics of the relevant states and associated excited state decay processes.

sexithiophene]-5-carboxylic acid or T6, Fig. 1). In this system, only the triplet energy transfer from the CdSe QD to T6 is energetically favored while the charge and singlet transfer are energetically uphill. We used transient absorption (TA) spectroscopy to first identify the lowest energy singlet $\left(\mathrm{S}_{1}\right)$ and triplet $\left(\mathrm{T}_{1}\right)$ state spectral features of free T6 molecules in solution. Then TA study of QDT6 complexes provide direct evidence for triplet sensitization of T6 by CdSe QD. We also employed Density Function Theory (DFT) computations to characterize the low-lying excited states involved in the triplet-triplet transfer mechanism.

T6 (shown in Fig. 1) was synthesized following a literature procedure ${ }^{28,29}$ and characterized by ${ }^{1} \mathrm{H}-\mathrm{NMR},{ }^{13} \mathrm{C}-\mathrm{NMR}$ and high resolution mass spectrometry (see ESI and Fig. $\mathrm{S} 1-\mathrm{S} 3 \uparrow$ for details). The absorption spectra of four QD-T6 complex samples with increasing T6 concentrations ranging from 12.5 to $50 \mu \mathrm{M}$ are shown in Fig. 2. These plots show a T6 band at $415 \mathrm{~nm}$ and the first excitonic peak of CdSe QDs at $584 \mathrm{~nm}$. In contrast to the absorption spectrum of T6 in toluene (Fig. 2 and S4 $\dagger$ ), the QD-

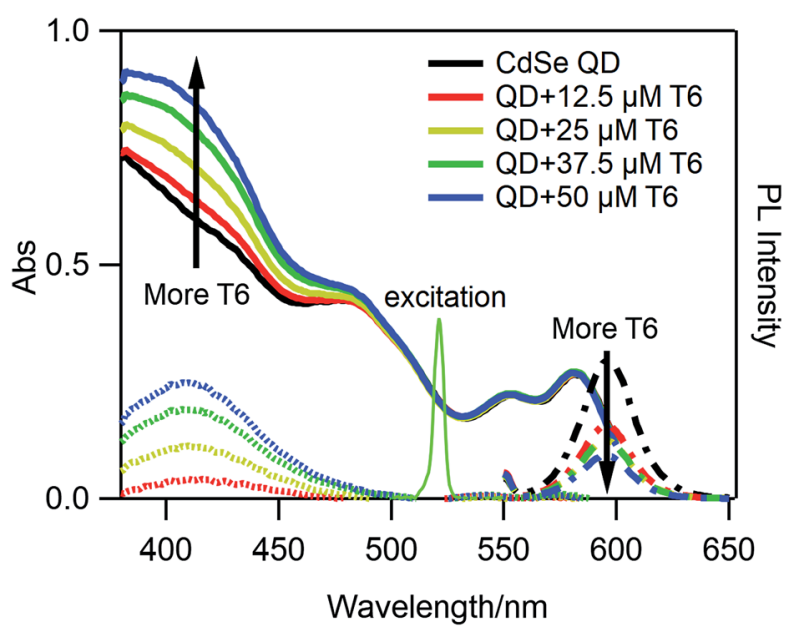

Fig. 2 UV-Vis absorption (solid lines) and emission (dash-dot lines) spectra of QD-T6 samples with increasing T6 concentrations ( 0 to 50 $\mu M)$ in toluene. The emission spectra were measured with $520 \mathrm{~nm}$ excitation of the QDs. Black lines are QD samples without T6. The adsorbed T6 UV-Vis absorption spectra are shown in dashed lines. The excitation pulse used in the transient spectroscopy study is also shown here. The $520 \mathrm{~nm}$ pulse has no overlap with T6 absorbance. bound T6 shows a red-shift of $\sim 20 \mathrm{~nm}$, which is attributed to a change in the dielectric environment and has been observed in a similar system. ${ }^{30}$ The total amounts of T6 in the samples were obtained by subtracting QD only spectra from QD-T6 complex spectra. However, the total T6 amount contains both QD-adsorbed and free T6 molecules, which cannot be easily differentiated by their absorption spectra alone. The amount of QD-bound T6 is determined by fitting the transient kinetics of triplet formation in QD-T6 complexes (see below). QD photoluminescence (PL) intensity decreased with increasing T6 concentration (Fig. 2), suggesting possible energy or charge transfer from the QD to T6.

To understand the fluorescence quenching mechanism in QD-T6 complexes, we studied pristine T6 first to characterize its excited states spectral signature via TA spectroscopy. The TA spectra of T6 measured with $400 \mathrm{~nm}$ excitation (Fig. 3A and B) show clear evolution from a singlet to triplet excited state on the sub-nanosecond time scale. The TA spectra at $<0.5 \mathrm{~ns}$ (Fig. 3A) show: (i) a ground $\left(\mathrm{S}_{0}\right)$ state bleach $(\mathrm{GSB})$ centered at $400 \mathrm{~nm}$ caused by the decrease of ground state T6 molecules; (ii) a stimulated emission from the T6 singlet excited state appearing as a negative signal at $500-600 \mathrm{~nm}$; and (iii) a broad singlet $\left(\mathrm{S}_{1}\right)$ excited state absorption (ESA) from $600 \mathrm{~nm}$ to $900 \mathrm{~nm}$. The assignment of GSB and stimulated emission of T6 is based on comparison with the steady state absorption and emission spectra shown in Fig. S4. $\dagger$ The $\left(\mathrm{S}_{1}\right)$ ESA peak is formed at an early delay time (2-5 ps) and its amplitude grows from $2-5$ ps (red) to $10-50$ ps (yellow) as the stimulated emission band shifts to longer wavelength (by $20 \mathrm{~nm}$ ) at the same time, which is attributed to a fast relaxation from the initial excited state to the $v=0$ vibrational level of the $\mathrm{S}_{1}$ excited state. Similar fast relaxation processes have also been observed in other oligothiophene molecules. ${ }^{26,31-34}$ Both the $S_{1}$ ESA and stimulated emission decay on the sub-nanosecond time scale to form the first triplet excited state $\left(\mathrm{T}_{1}\right)$ with an absorption peak $\left(\mathrm{T}_{1}\right.$ to $\mathrm{T}_{n}$ transition) centered at $715 \mathrm{~nm}$ (Fig. 3B). The lifetime of this species is longer than $10 \mu$ s and can be shortened by more than 1000 times in the presence of oxygen (Fig. S7 $\dagger$ ), consistent with the assignment to a $\mathrm{T}_{1}$ state. $^{35}$

We conducted DFT computations to determine the triplet excited state energetics on a model of the T6 molecule by
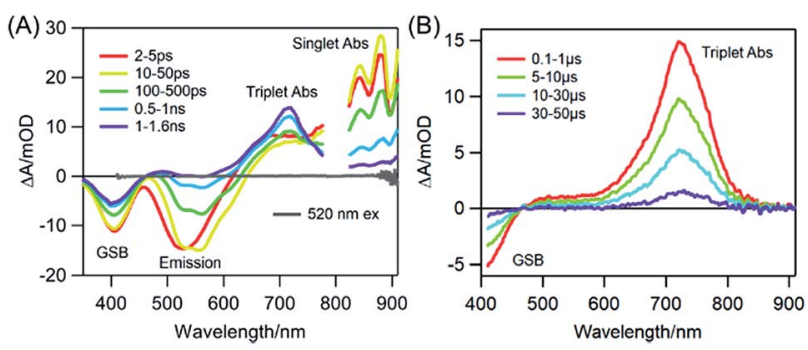

Fig. 3 TA spectra and kinetics of T6 in toluene. TA spectra of T6 at indicated delay times after $500 \mu \mathrm{J} \mathrm{cm}^{-2} 400 \mathrm{~nm}$ excitation: (A) 0-1.6 $\mathrm{ns}$ and (B) $0.1-50 \mu \mathrm{s}$. Data between $780-820 \mathrm{~nm}$ in (A) are cut out due to saturated probe light. $520 \mathrm{~nm}$ at $1 \mathrm{~mJ} \mathrm{~cm}^{-2}$ could not directly excite free $\mathrm{T} 6$ as shown in panel A (grey lines, averaged from 2 ps to $1 \mathrm{~ns}$ ). 
replacing the $\mathrm{C}_{6} \mathrm{H}_{13}$ alkyl side chains with methyl groups. All DFT computations were performed with the ORCA ${ }^{36}$ and Psi $4{ }^{37}$ programs using the $\mathrm{B}^{2} \mathrm{LYP}^{38}$ functional and the def2-SVP basis set. ${ }^{39,40}$ Equilibrium geometries for the $S_{0}$ and $T_{1}$ states are shown in Fig. S10 and S11. $\dagger$ From these geometries, we computed vertical excitation energies to higher states by timedependent density functional theory (TD-DFT) to identify lowlying electronic excited states involved in the triplet-triplet transfer mechanism. At the $\mathrm{S}_{0}$ geometry, $\mathrm{T}_{1}$ lies $1.85 \mathrm{eV}$ above the ground state and is characterized by a spin-flip HOMO $\rightarrow$ LUMO excitation. Geometric relaxation of the $T_{1}$ state stabilizes it by $0.47 \mathrm{eV}$, giving an adiabatic $\mathrm{S}_{0}$ to $\mathrm{T}_{1}$ transition energy of $1.38 \mathrm{eV}$. The second triplet state corresponds to a HOMO $\rightarrow$ LUMO + 1 excitation, with vertical transition energy of $2.10 \mathrm{eV}$. The third and fourth triplet states are predicted to lie at higher energies (2.64 and $2.82 \mathrm{eV}$ ) and therefore should not be operative in the observed triplet-triplet energy transfer.

To compute oscillator strengths for the $\mathrm{T}_{1} \rightarrow \mathrm{T}_{n}$ transitions, we performed a TD-DFT calculation at the optimized geometry of the $T_{1}$ state using the triplet state as a reference. The vertical excitation spectrum of $\mathrm{T}_{1}$ is dominated by a transition at $1.88 \mathrm{eV}$ $\left(659 \mathrm{~nm}, f_{\mathrm{osc}}=3.70\right)$ with a less intense peak at $2.18 \mathrm{eV}(568 \mathrm{~nm}$, $\left.f_{\text {osc }}=0.24\right)$. These results are in good agreement with the experimental observation of a transient peak at $715 \mathrm{~nm}$ in the transient absorption spectra for $t>100 \mathrm{~ns}$.

The mechanism of exciton quenching in CdSe QD/T6 complexes were then studied by transient absorption spectroscopy, which probes directly not only the quenching kinetics, but also the identity of intermediates and products. The TA spectra of the QD-T6 complexes at early delay times (1$10 \mathrm{ps}$ ) show that the selective excitation of CdSe QDs at $520 \mathrm{~nm}$ (where T6 does not absorb) leads to a QD ground state bleach and excited state absorption ${ }^{41-44}$ at 420-620 nm (Fig. 4A), similar to those observed in QD only samples (Fig. S5†) and indicative of excitons in the QD. The TA spectra at later delay times (Fig. 4A) show the decay of the QD exciton and the formation of a new absorption peak at 640-800 nm (Fig. S8d $\dagger$ for all T6 concentration) and $\mathrm{S}_{0}$ ground state bleach (Fig. 4B). These features are absent in QD only samples and can be assigned to $\mathrm{T} 6$ triplet formation by comparing to the T6 triplet TA spectrum (Fig. 4B). Control experiment of T6 only samples at $520 \mathrm{~nm}$ excitation (the grey circle in Fig. 4D, grey lines in Fig. 3A) shows negligible T6 triplet signal in the absence of QDs. Here the TA spectra data directly proved a successful triplet sensitization of T6 by QD.

To determine the mechanism of triplet formation, we compare the kinetics of T6 triplet state growth and exciton decay in QDs. The kinetics of triplet formation (Fig. 4C) can be monitored by both the triplet ESA (TF, blue circle) and $\mathrm{S}_{0}$ ground state bleach (GSB, black square). Here, triplet ESA kinetics was obtained by averaging TA signals from $650 \mathrm{~nm}$ to $780 \mathrm{~nm}$ to increase the signal/noise ratio. The T6 GSB is obtained by subtracting the overlapping QD contribution at $415 \mathrm{~nm}$ from the total signal. As reported previously, the CdSe $\mathrm{XB}$ signal is dominated by state filling of electrons in the conduction band edge. ${ }^{41-45}$ As shown in Fig. 4C, the triplet formation kinetics agree well with the exciton bleach $(\mathrm{XB}$, blue
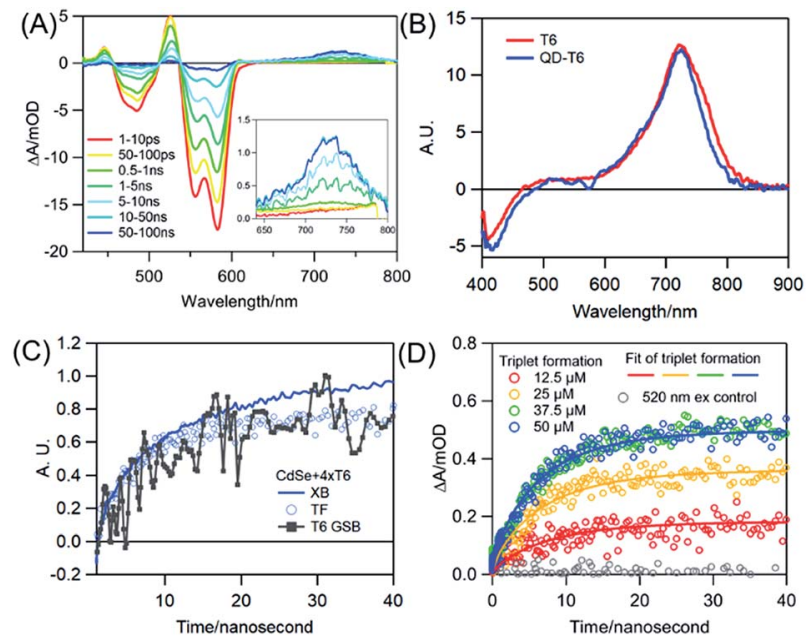

Fig. 4 Transient spectra and kinetics of QD/T6 complexes measured at $520 \mathrm{~nm}$ excitation. (A) TA spectra of QD-T6 excited at indicated delay times. Inset: expanded view of the triplet spectra at 540$800 \mathrm{~nm}$. (B) Comparison of the averaged TA spectra (from $100 \mathrm{~ns}$ to 5 $\mu \mathrm{s}$ ) of QD-T6 complexes (excited at $520 \mathrm{~nm}$ ) and pristine T6 (excited at $400 \mathrm{~nm}$ ). (C) Comparison of the kinetics of triplet formation of T6 (715 nm, blue circle), T6 GSB (415 nm, grey square, inverted and scaled) and QD exciton bleach (584 nm, blue line, inverted and scaled) in QDT6 complexes. (D) Kinetics of T6 triplet formation (TF) with increasing T6 concentration (red, yellow, green, blue) in QD-T6 complexes. Solid lines: fits according to a model described in the main text. Grey dots: T6 signal of a control sample with $50 \mu \mathrm{M}$ T6 and no QD measured under same condition at $520 \mathrm{~nm}$ excitation, showing no triplet signal. Excitation pulse energy density is $35 \mu \mathrm{J} \mathrm{cm}^{-2}$ for panel $A, C$ and D, 500 $\mu \mathrm{J} \mathrm{cm}^{-2}$ for panel $B$.

line) up to $\sim 20 \mathrm{~ns}$, consistent with a direct Dexter type TET from the QD to T6. These kinetics deviate after $\sim 20 \mathrm{~ns}$, which suggests the presence of other exciton decay pathways. As discussed below, we attribute this to the presence of QDs without adsorbed T6, in which excitons decay by radiative and nonradiative pathways within the QD. It has been previously reported that QD sensitized triplet formation can also occur via sequential charge transfer pathways: electron (hole) transfer followed by hole (electron) transfer. ${ }^{4-48}$ These pathways would produce $\mathrm{T}^{+}$or $\mathrm{T}^{-}$intermediates, which are not observed in our study. This observation is also supported by the energetics alignment that prohibits charge transfer or singlet energy transfer (Fig. S12 $\dagger$ ). Therefore, we attribute this sensitization process to be a direct Dexter-type triplet energy transfer from a triplet exciton state in the QD.

To examine whether TET occurs via static or dynamic quenching mechanisms, we measure TET kinetics as a function of total T6 concentration in the sample. As shown in Fig. 4D, the final amplitude and apparent formation rate of the T6 triplet state increase with the total T6 concentration, until it reaches saturation. Similar acceptor concentration dependent kinetics has been observed in previous studies of QD/electron acceptor complexes. ${ }^{\mathbf{4 4 , 4 9 - 5 2}}$ This kinetics can be well described by a model that assumes that the number of adsorbed acceptor molecules in $\mathrm{QD} /$ acceptor complexes follows a Poisson distribution and the rate of transfer is proportional to the number of adsorbed 
acceptors. Assuming the same model for TET in QD/acceptor complexes, we can derive an expression of the kinetics of triplet formation (eqn (2)) and QD exciton bleach recovery (see ESI $\dagger$ ).

$$
[\mathrm{T} 6]_{\mathrm{T}}^{*}(t)=[\mathrm{QD}(0)]^{*} \sum_{i} P(m, i) \sum_{j} a_{j} i k_{\mathrm{TET}}\left(1-\frac{\mathrm{e}^{-\left(k_{1 j}+i k_{\mathrm{TET}}\right) t}}{k_{1 j}+i k_{\mathrm{TET}}}\right)
$$

In eqn (2), $P(m, i)$ is the Poisson distribution probability of finding QD/T6 complexes with $i$ adsorbed T6 molecules in a sample with $m$ average number of acceptors. $k_{\text {TET }}$ is the intrinsic energy transfer rate in a $1: 1 \mathrm{QD} / \mathrm{T} 6$ complex. The decay of QD exciton bleach through radiative and nonradiative pathways within the QD is described by multiple exponential decay with amplitude $a_{j}$ and rate constant $k_{1 j}$. [QD(0) $]^{*}$ is the initial concentration of excited QDs. Further details of the model and derivation can be found in the ESI. $\dagger$ Because the decay rate of T6 triplet excited state $\left(k_{3}\right)$ is three orders of magnitude slower than triplet formation (Fig. S7 $\dagger$ ), it is not considered in the fitting of the triplet formation kinetics. The T6 triplet formation (Fig. 4D) and QD exciton bleach recovery (Fig. S8a $\dagger$ ) can be well fit by this model with an intrinsic TET of $0.077 \pm 0.002 \mathrm{~ns}^{-1}$. The fit also yields the average number of adsorbed T6 molecules as a function of total T6 concentration, ranging from 0.16 to 0.54 . This dependence can be fit to a Langmuir isotherm, as shown in Fig. S8b, $\uparrow$ to obtain a maximum number of adsorption sites and binding constant of T6 on CdSe QDs of $\theta=1.4 \pm 0.8$, and $K=0.014 \pm 0.012 \mu \mathrm{M}^{-1}$, respectively. It is important to note that in the range of $m$ (from 0.16 to 0.54 ) studied in this work, over $90 \%$ of the QD population in the samples has zero or one adsorbed T6 (Table S2 $\dagger$ ) and the triplet formation kinetics is mostly contributed by QD with one bounded T6. This is confirmed by the comparison of normalized triplet formation kinetics for different samples (Fig. S8c $\dagger$ ), which are identical within the $\mathrm{S} / \mathrm{N}$ ratio of this measurement. The increased T6 signal amplitude in Fig. 4D reflects a larger percentage of $\mathrm{T} 6$ bounded QDs in these samples.

From the relative amplitude of the triplet state absorption and QD exciton bleach and their extinction coefficients, we can estimate a TET efficiency of $\mathbf{1 5 . 4} \pm \mathbf{0 . 6 \%}$ in the sample with 50 $\mu \mathrm{M}$ T6 concentration (see ESI $\dagger$ for details). The low efficiency can be attributed to both the lower number of adsorbed T6 molecules and slow intrinsic TET rate. The average number of adsorbed T6 per QD is only around 0.5 at the highest coverage level, presumably limited by the competitive adsorption between T6 and the stabilization oleic acid ligand on the QD surface. In comparison, the Castellano group reported CdSe QD to 9-anthracene carboxylic acid (ACA) Dexter TET with an overall rate of $2 \times 10^{9} \mathrm{~s}^{-1}$ with an average number of ACA per QD of 12. ${ }^{12}$ This yields a similar intrinsic TET rate constant of 0.17 $\mathrm{ns}^{-1}$. To verify this point, we vigorously sonicated the sample with excess amount of T6 $(375 \mu \mathrm{M})$ and measured the TA spectrum within an hour. This temporarily created a high loading which leads to more efficient TET at $31.8 \pm 1.2 \%$ as shown in Fig. S9. $\dagger$ Thus, a promising approach to increase the overall TET rate and efficiency in the T6/QD complex is to improve the binding strength of T6, through perhaps a different anchoring group.

\section{Conclusions}

We have demonstrated the first example of triplet energy sensitization of an oligothiophene (T6) by CdSe QDs. By TA spectroscopy we confirmed this process occur via direct Dextertype triplet energy transfer. The efficiency of TET increases with the concentration of T6 in solution, reaching a value of $15.4 \pm$ $0.6 \%$ in the sample with $50 \mu \mathrm{M}$ T6 and $31.8 \pm 1.2 \%$ in the sample with $375 \mu \mathrm{M}$ of T6, although the latter exhibits poor long-term stability. The T6 concentration-dependent TET kinetics can be understood by a model that assumes a Poisson distribution of adsorbed T6 molecule on the QD. This analysis suggests that TET rates are similar in the $1: 1$ QD-T6 and previously reported QD-anthracene complexes, but T6 acceptors have relatively weaker binding constant on the surface, which leads to a smaller average number of acceptors and slower apparent TET rate. Our finding suggests that TET efficiency QDT6 complexes can likely be further improved by increasing the acceptor binding strength (through modification of the acceptor and QD surface ligands) and fine-tuning energetic levels. Finally, this work identifies a new class of stable and highly tunable/functionalizable organic TET acceptors.

\section{Conflicts of interest}

There are no conflicts to declare.

\section{Acknowledgements}

T. L. gratefully acknowledges the financial support from the National Science Foundation (CHE-1709182). E. A. acknowledges support from the National Science Foundation (CHE1821863). F. A. E. acknowledges support from the U.S. Department of Energy under Award No. DE-SC0016004, the Camille and Henry Dreyfus Foundation under award TC-18-045, and the Alfred P. Sloan Foundation under award FG_20166748.

\section{Notes and references}

1 C. J. Bardeen, Annu. Rev. Phys. Chem., 2014, 65, 127-148.

2 A. Köhler and H. Bässler, J. Mater. Chem., 2011, 21, 40034011.

3 A. Köhler and H. Bässler, Mater. Sci. Eng., R, 2009, 66, 71-109. 4 T. N. Singh-Rachford and F. N. Castellano, Coord. Chem. Rev., 2010, 254, 2560-2573.

5 J. de Wild, A. Meijerink, J. K. Rath, W. G. J. H. M. van Sark and R. E. I. Schropp, Energy Environ. Sci., 2011, 4, 4835-4848.

6 W. Shockley and H. J. Queisser, J. Appl. Phys., 1961, 32, 510519.

7 J. Peng, X. Guo, X. Jiang, D. Zhao and Y. Ma, Chem. Sci., 2016, 7, 1233-1237.

8 T. C. Wu, D. N. Congreve and M. A. Baldo, Appl. Phys. Lett., 2015, 107, 031103. 
9 N. Yanai, M. Kozue, S. Amemori, R. Kabe, C. Adachi and N. Kimizuka, J. Mater. Chem. C, 2016, 4, 6447-6451.

10 N. Yanai and N. Kimizuka, Acc. Chem. Res., 2017, 50, 24872495.

11 Z. Huang, X. Li, M. Mahboub, K. M. Hanson, V. M. Nichols, H. Le, M. L. Tang and C. J. Bardeen, Nano Lett., 2015, 15, 5552-5557.

12 C. Mongin, S. Garakyaraghi, N. Razgoniaeva, M. Zamkov and F. N. Castellano, Science, 2016, 351, 369-372.

13 M. Wu, D. N. Congreve, M. W. B. Wilson, J. Jean, N. Geva, M. Welborn, T. Van Voorhis, V. Bulović, M. G. Bawendi and M. A. Baldo, Nat. Photonics, 2016, 10, 31-34.

14 K. Okumura, K. Mase, N. Yanai and N. Kimizuka, Chem.-Eur. J., 2016, 22, 7721-7726.

15 W. W. Yu, L. Qu, W. Guo and X. Peng, Chem. Mater., 2003, 15, 2854-2860.

16 G. D. Scholes and G. Rumbles, Nat. Mater., 2006, 5, 683-696. 17 J. Jasieniak, M. Califano and S. E. Watkins, ACS Nano, 2011, 5, 5888-5902.

18 Z. Huang, X. Li, B. D. Yip, J. M. Rubalcava, C. J. Bardeen and M. L. Tang, Chem. Mater., 2015, 27, 7503-7507.

19 M. Mahboub, Z. Huang and M. L. Tang, Nano Lett., 2016, 16, 7169-7175.

20 V. Gray, P. Xia, Z. Huang, E. Moses, A. Fast, D. A. Fishman, V. I. Vullev, M. Abrahamsson, K. Moth-Poulsen and M. Lee Tang, Chem. Sci., 2017, 8, 5488-5496.

21 M. Mahboub, H. Maghsoudiganjeh, A. M. Pham, Z. Huang and M. L. Tang, Adv. Funct. Mater., 2016, 26, 6091-6097.

22 Z. Huang, D. E. Simpson, M. Mahboub, X. Li and M. L. Tang, Chem. Sci., 2016, 7, 4101-4104.

23 D. L. J. Dexter, A Theory of Sensitized Luminescence in Solid, 1953.

24 Z. Huang and M. L. Tang, J. Am. Chem. Soc., 2017, 139, 94129418.

25 A. R. Reddy and M. Bendikov, Chem. Commun., 2006, 11791181, DOI: 10.1039/b513597d.

26 R. S. Becker, J. S. deMelo, A. L. Macanita and F. Elisei, J. Phys. Chem., 1996, 100, 18683-18695.

27 R. Stalder, D. Xie, R. Zhou, J. Xue, J. R. Reynolds and K. S. Schanze, Chem. Mater., 2012, 24, 3143-3152.

28 S. Dufresne, G. S. Hanan and W. G. Skene, J. Phys. Chem. B, 2007, 111, 11407-11418.

29 Y. Huang, Z. Xu, S. Jin, C. Li, K. Warncke, F. A. Evangelista, T. Lian and E. Egap, Chem. Mater., 2018, 30, 7840-7851.

30 Z. Huang, Z. Xu, M. Mahboub, X. Li, J. W. Taylor, W. H. Harman, T. Lian and M. L. Tang, Angew. Chem., 2017, 129, 16810-16814.

31 R. S. Becker, J. S. Demelo, A. L. Macanita and F. Elisei, Pure Appl. Chem., 1995, 67, 9-16.
32 J. P. Yang, W. Paa and S. Rentsch, Chem. Phys. Lett., 2000, 320, 665-672.

33 J. P. Yang, W. Paa and S. Rentsch, Synth. Met., 1999, 101, 624-625.

34 D. V. Lap, D. Grebner and S. Rentsch, J. Phys. Chem. A, 1997, 101, 107-112.

35 C. Grewer and H.-D. Brauer, J. Phys. Chem., 1994, 98, 42304235.

36 F. Neese, Wiley Interdiscip. Rev.: Comput. Mol. Sci., 2012, 2, 73-78.

37 R. M. Parrish, L. A. Burns, D. G. A. Smith, A. C. Simmonett, A. E. DePrince, E. G. Hohenstein, U. Bozkaya, A. Y. Sokolov, R. Di Remigio, R. M. Richard, J. F. Gonthier, A. M. James, H. R. McAlexander, A. Kumar, M. Saitow, X. Wang, B. P. Pritchard, V. Prakash, H. F. Schaefer, K. Patkowski, R. A. King, E. F. Valeev, F. A. Evangelista, J. M. Turney, T. D. Crawford and D. Sherrill, J. Chem. Theory Comput., 2017, 13, 3185-3197.

38 A. D. Becke, J. Chem. Phys., 1993, 98, 5648-5652.

39 F. Weigend and R. Ahlrichs, Phys. Chem. Chem. Phys., 2005, 7, 3297-3305.

40 F. Weigend, J. Comput. Chem., 2008, 29, 167-175.

41 J. Q. Chen, K. F. Wu, B. Rudshteyn, Y. Y. Jia, W. D. Ding, Z. X. Xie, V. S. Batista and T. Q. Lian, J. Am. Chem. Soc., 2016, 138, 884-892.

42 H. Zhu, N. Song and T. Lian, J. Am. Chem. Soc., 2010, 132, 15038-15045.

43 J. Huang, Z. Huang, Y. Yang, H. Zhu and T. Lian, J. Am. Chem. Soc., 2010, 132, 4858-4864.

44 J. Huang, D. Stockwell, Z. Huang, D. L. Mohler and T. Lian, J. Am. Chem. Soc., 2008, 130, 5632-5633.

45 V. I. Klimov, Annu. Rev. Phys. Chem., 2007, 58, 635-673.

46 S. Garakyaraghi, C. Mongin, D. B. Granger, J. E. Anthony and F. N. Castellano, J. Phys. Chem. Lett., 2017, 8, 1458-1463.

47 S. S. Skourtis, C. Liu, P. Antoniou, A. M. Virshup and D. N. Beratan, Proc. Natl. Acad. Sci. U. S. A., 2016, 113, 8115-8120.

48 D. J. Weinberg, S. M. Dyar, Z. Khademi, M. Malicki, S. R. Marder, M. R. Wasielewski and E. A. Weiss, J. Am. Chem. Soc., 2014, 136, 14513-14518.

49 A. Boulesbaa, A. Issac, D. Stockwell, Z. Huang, J. Huang, J. Guo and T. Lian, J. Am. Chem. Soc., 2007, 129, 1513215133.

50 M. Tagliazucchi, D. B. Tice, C. M. Sweeney, A. J. MorrisCohen and E. A. Weiss, ACS Nano, 2011, 5, 9907-9917.

51 A. Issac, S. Jin and T. Lian, J. Am. Chem. Soc., 2008, 130, 11280-11281.

52 S. Jin, J. C. Hsiang, H. Zhu, N. Song, R. M. Dickson and T. Lian, Chem. Sci., 2010, 1, 519-526. 